\section{Laparoscopic-Assisted Appendicectomy (LAA): \\ A Novel Advance on an Established Procedure}

Laparoscopic appendicectomy has been shown to be a safe alternative to open appendicectomy [1-3]. However some inherent disadvantages of this procedure have prohibited its universal acceptability [4]. We have devised a "two-port modified technique" (Figure 1) for laparoscopicassisted appendicectomy (LAA) which we believe addresses these problems.

A 12-mm umbilical port is inserted using the Hasan technique. Pneumoperitoneum is achieved, and the laparoscopic camera inserted. The patient is placed in the Trendelenburg position with a $15^{\circ}$ tilt to the left side. A $10-\mathrm{mm}$ port is inserted near the McBurney point, guided by the site of the appendix or cecum, under direct vision. The appendix is identified and the diagnosis of appendicitis is established. The appendix is grasped with a nontraumatic grasper, from the distal mesentery in line with the grasper, and pulled into the port for some distance. The grasper holding the appendix and the port are delivered into the port site. The mesentery is grasped with a Babcock forceps and the port and nontraumatic grasper removed. Appendicectomy is carried out in the conventional fashion, by gradually delivering the whole of the appendix (Figure 2). The cecum is dropped back into the peritoneal cavity. The cecum and stump are visualized using the camera, for checking homeostasis and security. The muscles at the site of the right iliac fossa port rarely need a suture for closure.

The disadvantages of laparoscopic appendicectomy have been longer operating time [1-3] and high cost [4]. LAA inherits the advantages of both laparoscopic and open appendicectomy. It can be performed in most cases, except where the appendix is very friable. We have carried out the procedure in 26 patients without complications. The theoretical possibility of wound infection is reduced by careful handling of the appendix, minimizing its contact with the wound.

A similar technique has been described previously; however in that technique, ligation of the appendicular vessels is done inside the peritoneal cavity [5]. We exteriorize the appendix with intact mesentery and complete the procedure in a conventional manner. Because of the limited intraperitoneal component of the laparo-

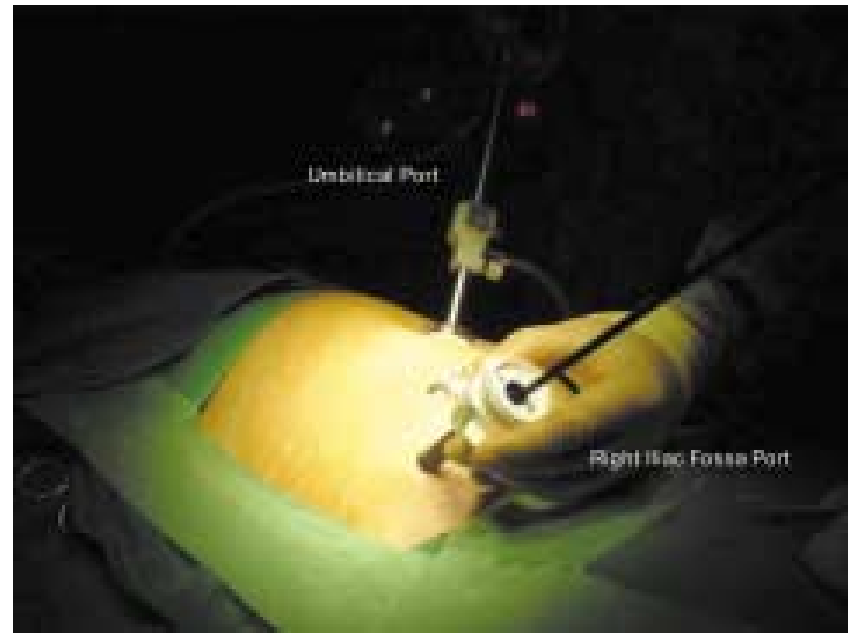

Figure 1 Placement of ports for laparoscopic-assisted appendicectomy

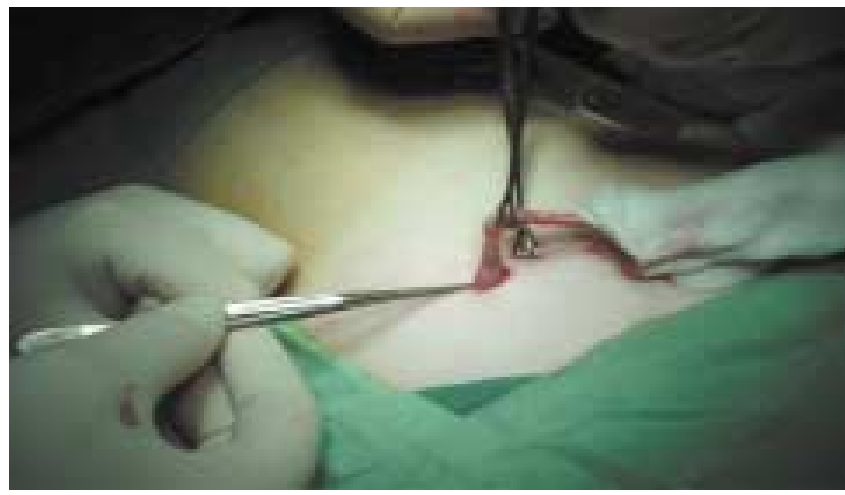

Figure 2 The appendix is delivered through the port-site incision. The mesoappendix and vessels are ligated and divided, as is the appendix base

scopic procedure, LAA has a steeper learning curve. Extraperitoneal ligation of the appendix base and appendicular vessels reduces the operating time. LAA obviates the need for disposable laparoscopic instruments with the potential of reducing the costs associated with laparoscopic appendicectomy.

\section{Z. Khan, R. Mofidi, H. P. Redmond}

Department of Academic Surgery,

Cork University Hospital, Wilton,

Cork, Ireland

\section{References}

${ }^{1}$ Hellberg A, Rudberg C, Kullman E, et al. Prospective randomized multicentre study of laparoscopic versus open appendicectomy. Br J Surg 1999; 86 (1): $48-$ 53

${ }^{2}$ Reiertsen O, Larsen S, Trondsen E, et al. Randomized controlled trial with sequential design of laparoscopic versus conventional appendicectomy. Br J Surg 1997; 84 (6): $842-847$

${ }^{3}$ Tate JJ, Chung SC, Dawson J, et al. Laparoscopic versus open appendicectomy: prospective randomised trial. Lancet 1993; 342 (8872): 633-637
${ }^{4}$ Kald A, Kullman E, Anderberg B, et al. Cost-minimisation analysis of laparoscopic and open appendicectomy. Eur J Surg 1999; 165 (6): 579-582

${ }^{5}$ Goh P, Tekant Y, Kum CK, et al. Technical modification to laparoscopic appendectomy. Dis Colon Rectum 1992; 35 (10): 999-1000

Corresponding Author

Z. Khan, FRCSI

Lecturer in Surgery

Cork University Hospital

117 Pace Road

Little Pace, Paddocks

Dublin 15

Ireland

Fax: +353-21-344230

E-mail: zaib@eircom.net 